\title{
The Specifics of Calculating the Efficiency of the Continuously Variable Mechanical Transmission with Oscillatory Movement of Internal Links and Force Function Control
}

\author{
AndreyYurkevich ${ }^{1, *}$, and Larisa Yurkevich ${ }^{2}$ \\ ${ }^{1}$ Institute of Engineering Science, Ural Branch of the Russian Academy of Sciences, 34, \\ Komsomolskaya St., 620049, Ekaterinburg, Russia \\ ${ }^{2}$ Kurgan State University, Bldg. 4, 63, Sovetskaya St., Kurgan, 640020, Russia
}

\begin{abstract}
The paper discusses the energy flow in the continuously variable mechanical transmission with oscillatory movement of internal links and force function control. It is proposed to treat the potential energy of the twisted torsion bars, which is transferred back to the drive shaft, as useful. The formula for calculating the transmission efficiency, taking into account the return of energy flow back to the motor, hysteresis losses, and energy dissipation in the mechanical rectifier while taking up the free play, is given. When calculating the efficiency of the continuously variable mechanical transmission, it is suggested to take into consideration the clearances in the rectifier and kinematic pairs using 'kinematic efficiency'. The examples of measured and calculated transmission efficiency in the 'stop' mode are given.
\end{abstract}

\section{Introduction}

Traditionally, the vehicle transmission efficiency is estimated by the efficiency factor. In such a case, the efficiency is defined as the ratio of the useful power $N_{2}$ on the transmission driven shaft to the supplied power $N_{1}$ on the drive shaft.

$$
\eta=N_{2} / N_{1}=M_{2} \omega_{2} / M_{1} \omega_{1}
$$

where $M_{2}, M_{1}$ - the torques in the driven and drive shafts of the transmission, respectively;

$\omega_{2}, \omega_{1}$ - angular velocity of the driven and drive shafts, respectively.

As it applies to fixed-ratio mechanical transmissions, there is a rigid kinematic constraint between the drive and driven shafts. The transmission efficiency depends on the kinematic scheme and the torque losses caused by gear face friction. Friction creates thermal energy that cannot be fully converted back to work, so it is not recoverable. Energy

*Corresponding author: urkeva@mail.ru 
is transferred only in one direction from the internal combustion engine to the transmission driven shaft.

Self-regulating continuously variable hydrodynamic transmissions [1] have a 'stop' mode $\left(\omega_{2}=0\right.$ at $\omega_{1} \neq 0$ ). In this mode, all the energy from the impeller dissipates as heat, so it is lost or not recoverable. In most cases, energy is transferred only in one direction from the drive motor to the driven shaft in such gears.

Differential hybrid transmission systems [2] have several shafts which can be both drive and driven, depending on the operating mode. This makes it possible to return excess energy from the internal combustion engine to electric machines, which can go to the generator mode with battery charging. In calculations, this is expressed in changing the sign in front of the efficiency value.

In the continuously variable mechanical transmission with oscillatory movement of internal links and force function control (the Blagonravov transmission) $[3,4,5]$ the output end of the driven shaft is stationary in the 'stop' mode $\left(i=\omega_{2} / \omega_{1}=0\right)$. Therefore, the potential energy of the twisted torsion bars, received from the ICE in one cycle, is transferred back to the drive shaft, reducing the amount of work done by the power source. That is the transmission's unique feature. The recovered energy can be treated as useful, since only the work that cannot be returned should be considered as expended energy [6]. Figure 1 presents dimensionless characteristic of this transmission [3].

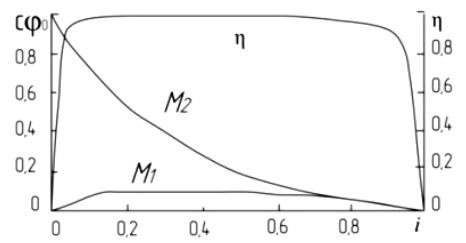

Fig. 1. Dimensionless characteristic of the Blagonravov continuously variable mechanical transmission [3]. $M_{2}, M_{1}$ - average cycle value of relative torques in the transmission driven and drive shafts, respectively; $c_{t}$ - the torsion shaft stiffness; $\varphi_{0}$ - the vibration amplitude of the rocker arms of mechanical rectifiers.

Therefore, the use of formula (1) to calculate the transmission efficiency ( $\eta$ ) does not provide sufficient information to estimate the energy losses. For example, in the 'stop' mode $i=\omega_{2} / \omega_{1}=0, \eta=0$, although the losses can be very low. The specifics of calculating the Blagonravov transmission efficiency is the subject of the present discussion. It should also be noted that the return of energy to the drive shaft also takes place in inertial automatic torque transformers $[7,8]$.

\section{The specifics of loading the ICE by continuously variable mechanical transmission}

The Blagonravov transmission operational guideline and control strategy are considered in sufficient detail in $[5,9]$. To calculate the transmission efficiency, let us consider the specifics of loading the internal combustion engine through the mechanics of energy delivery from the ICE shaft to the transmission driven shaft. Figure 2 demonstrates the diagram of one path of the transmission. Briefly, the principle of its operation is as follows.

The transmission drive shaft connected to the ICE 3 rotates at a constant speed $\omega_{1}$. With the help of the articulation linkage (crank $r$, crank rod $l_{1}$ ), rotation is converted into angular 
vibrations of the rocker arm $l_{2}$ of the mechanical rectifier 1 (free-wheeling mechanism FWM). A new reliable axial free-wheeling mechanism with intermediate rolling elements and additional working surfaces is used as a FWM $[10,11]$.

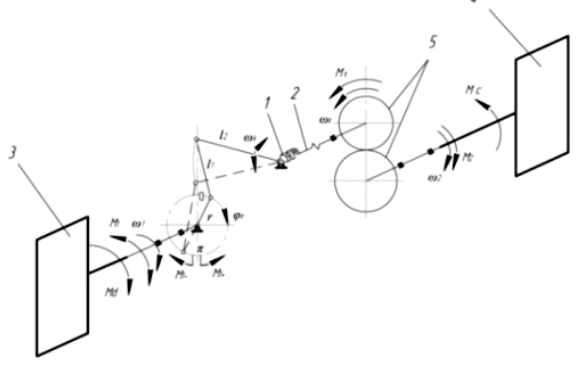

Fig. 2. One path of the transmission flow chart. 1 - FWM; 2 - torsion shaft; 3 - internal combustion engine (ICE); 4 - external load; 5 - gear wheels of the compound.

The rectifier 1 provides kinematic condition according to which the speed of its driven element connected to the input end of the torsion shaft 2 can be greater, but cannot be less than the angular velocity of the rocker arm $\omega_{k}$. The output end of the torsion shaft 2 is connected to the gear wheels 5 of the compound. The central wheel of this compound is the transmission driven shaft connected to an external load 4. The oscillation amplitude $\varphi_{0}$ of the rocker arm, being determined by the radius of the crank $r$, is given by the formula $\varphi_{0}=\arcsin \left(r / l_{2}\right)$. The mechanism for changing the amplitude is not presented here.

If the transmission driven shaft is stationary ('stop' mode $i=0$ ), then the rear ends of the torsion bars are also stationary $\omega_{t}=\omega_{2}=0$, and the forward ends, connected to the driven elements of the rectifiers, turn towards the power stroke of the rocker arm $\varphi_{r}$. In this case, the rectifiers are turned on during the entire revolution of the drive shaft. As a result, the torsion bars are twisted, their elastic deformation occurs. For this elastic deformation $\varphi_{t}$ the ICE energy is spent.

In the 'stop' mode, for the first half of the oscillation period $\omega_{1} t_{1}=0<\omega_{1} t<\pi,\left(t_{1}-\right.$ the time interval at which the rectifier wedges up) the input end of the torsion bar turns in the direction opposite to the torque $M_{1}^{+}$(see Fig. 2.), while the potential energy of the torsion bar increases from zero to maximum. In this case, the ICE torque $M_{d}$ performs work, which should be considered as the work input. For the second half of the oscillation period $\pi<\omega_{1} t<2 \pi=\omega_{1} t_{3}\left(t_{3}\right.$ - the time interval at which the rectifier wedges out) the direction of the rocker arm movement is reversed. In this case, the work output of the torque $M_{2}$ on the transmission driven shaft is equal to zero, since $M_{2}<M_{c}$ and $\omega_{t}=\omega_{2}=0$.

The potential energy of the twisted torsion bar will be consumed only from the input end and return to the drive shaft through the oscillation converter [5]. This energy can be regarded as useful. The direction of the torque $M_{1}^{-}$(see Fig. 2) coincides with $M_{d}$ decreasing its value. The average amount of work done by the torque $M_{1}^{-}$is less than that done by the torque $M_{1}^{+}$by the amount of hysteresis losses in the rectifier [11], as well as by the amount of losses in the transmission kinematic pairs. In this case, the motor torque remains the same; it is needed only to cope with the frictional losses in the loaded converter. These processes are discussed in more detail in [5]. 
The kinematic and force relations allow the work input and output to be quantified per cycle and to draw up a calculated efficiency formula for such a transmission. Here, as in [3], the following assumptions are adopted: the output end of the torsion shaft rotates with $\omega_{2}=$ const; the rocker arm of the mechanical rectifier executes a harmonic vibration; the rectifiers are switched on and off without frictional sliding of their driven and drive elements. Then

$$
\begin{gathered}
\varphi_{r}=\omega_{1} t, \\
\varphi_{k}=\varphi_{0}\left(1-\cos \left(\omega_{1} t\right)\right), \\
\omega_{k}=\varphi_{0} \omega_{1} \sin \left(\omega_{1} t\right), \\
\varphi_{2}=i \omega_{1} t, \\
\omega_{2}=i \omega_{1},
\end{gathered}
$$

where $t$-time; $\varphi_{2}$ - the angle of rotation of the output end of the torsion shaft; $i$ - the total gear ratio, which is given by the formula

$$
i=i_{\Pi} i_{t} i_{p}
$$

where $i_{\Pi}$ - maximum ratio of the oscillation generator [3] (for the harmonic law $i_{\Pi}-\varphi_{0}$; $i_{t}$ - internal gear ratio, varying from 0 to $1[3] ; i_{P}$ - gear ratio of the compound, for the given diagram $i_{p}-1$.

The rectifier is activated at the drive shaft phase angle $\omega_{1} t_{1}$, and it is switched off at the phase angle $\omega_{1} t_{3}$, at which the torsion $\varphi_{t}$ becomes equal to zero. According to [3], the angle of rotation $\omega_{1} t_{1}=\arcsin \left(i_{t}\right)$, and $\omega_{1} t_{3}$ is given by the transcendental equation

$$
\cos \left(\omega_{1} t_{1}\right)-\cos \left(\omega_{1} t_{3}\right)-i_{t} \omega_{1}\left(t_{3}-t_{1}\right) .
$$

Let us assume that the stiffness of the rectifier is significantly higher than the stiffness of the torsion bar and is not taken into account in the stiffness analysis, as it is generally regarded in [3]. Then the torsion is given as $\varphi_{t}=\varphi_{k}-\varphi_{2},(3,5$ and 7 are taken into account):

$$
\varphi_{t}=\varphi_{0}\left[\cos \left(\omega_{1} t_{1}\right)-\cos \left(\omega_{1} t\right)-i_{t}\left(t-t_{1}\right)\right]
$$

Using constraint equation for the angular deformations and torsion stiffness $c_{t}$, the value of the torque twisting the torsion bar can be calculated as

$$
M_{2}=c_{t} \varphi_{t}
$$

The value of the torque loading the engine is determined taking into account the ratio between the rocker arm and the drive shaft $\omega_{k} / \omega_{1}=\varphi_{0} \sin \left(\omega_{1} t\right)$ by eq.

$$
M_{d}=M_{1} \varphi_{0} \sin \left(\omega_{1} t\right) M_{2}
$$

In fig. 3.a the results of calculating the kinematic parameters at various values of the internal gear ratio $i_{t}$ are given in relative units. Point $a$ corresponds to the rocker arm angle $\omega_{1} t_{3}$ at which the rectifier wedges out. In Fig. 3.b. the results of calculating $M_{1} / c_{t} \varphi_{0}$ according to (11) are presented. 
The work done by the torque $M_{1}^{+}$corresponds to the energy supplied by the ICE, and the work done by $M_{1}^{-}$corresponds to the potential energy of the twisted torsion bar returned to the engine that can be regarded as useful, but performed by the torsion bar. If the potential energy of the torsion bar returns to the drive shaft without losses while the unloading, then the rolling friction work of the balls filling the rectifier slots should be considered as hysteresis losses in the loading-unloading (handling) cycle and taken into account when calculating the efficiency. In this case, the torque in the drive shaft of the transmission will be greater than the torque required to cut the losses in the kinematic pairs of the converter by the amount of losses during loading and unloading of the rectifiers. The transmission is distinguished by the return of energy to the drive shaft of the ICE in certain operating modes. In similar continuously variable mechanical transmissions, but without torsion shafts [12], there is no energy recovery.

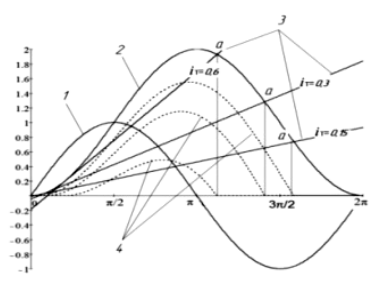

a

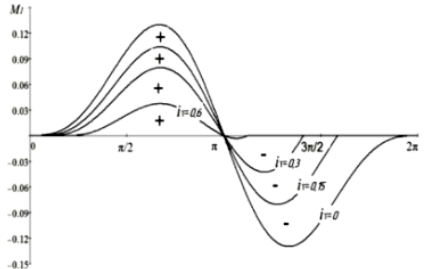

$\mathrm{b}$

Fig. 3. Kinematic and force parameters of one transmission path per cycle depending on the internal gear ratio $i_{t}$. a) kinematic parameters; b) the torque on the transmission drive shaft. $1-\omega_{k} / \varphi_{0} \omega_{1} ; 2$ $\varphi_{k} / \varphi_{0} ; 3-\varphi_{2} / \varphi_{0} ; 4-\varphi_{t} / \varphi_{0}$.

Another feature of the transmission is the presence of end clearances in the rectifiers and bottom clearances in the gear meshing of the transmission [3, 13], which negatively affects the magnitude of the angular velocity of the driven link. Due to the clearances the actual $\varphi_{0}^{*}$ vibration amplitude is less than the geometrically established one, $\varphi_{0}$. With that, the actual transmission characteristic differs from the theoretical one. It turns out the same as in the absence of clearances, but with the amplitude $\varphi_{0}^{*}$, reduced by the value $\Delta \varphi_{0}=\varphi_{0}-\varphi_{0}^{*}$ in the entire kinematic transmission chain [3]. In this case, the speed of the output shaft will be less than the theoretical one by the value of the kinematic backlash $\Delta n_{2}=n_{2}^{T}-n_{2}^{\ni}$, where $n_{2}^{T}$ is theoretically calculated angular velocity of the shaft; $n_{2}^{\ni}$ is the actual angular velocity of the shaft. Then, taking into account that $n_{2}^{T}=\varphi_{0} i_{t} i_{p} n_{1}{ }^{3}$ and $n_{2}^{\ni}=\varphi_{0}^{*} i_{t} i_{p} n_{1}^{\ni}$ can be given by formula $\Delta n_{2}=\left(\varphi_{0}-\varphi_{0}^{*}\right) i_{t} i_{p} n_{1}^{\ni}=\Delta \varphi_{0} i_{t} i_{p} n_{1}^{\ni} . \Delta \varphi_{0}$ can be taken as kinematic backlash, expressed in radians. In the absence of load on the output shaft $\left(M_{2}=0\right)$, the internal gear ratio $i_{t}=1$. This allows us to make adjustments to the geometrically set oscillation amplitude of the rectifier rocker arm $\varphi_{0}$ by the value of the angular clearances and determine:

$$
\begin{gathered}
\varphi_{0}^{*}=n_{2}^{\ni} / i_{p} n_{1}^{\ni}, \\
\Delta \varphi_{0}=\varphi_{0}-n_{2}^{\ni} / i_{p} n_{1}^{\ni} .
\end{gathered}
$$

Some experimental studies [14] on the driven shaft in the absence of load $\left(M_{2}=0\right)$ have been made. The results of calculating the backlash according to (13) vary from $5.5^{\circ}$ to $3.4^{\circ}$ and depend on the angular velocity of the input shaft. The test results show that the value of 
the backlash decreases [13] when the frequency of the input shaft rotation increases. This is due to the end clearance elimination by the half couplings in the rectifiers before wedging. Fig. 4 demonstrates experimental data from [14] at the set vibration amplitude $\varphi_{0}=0,172$ rad.

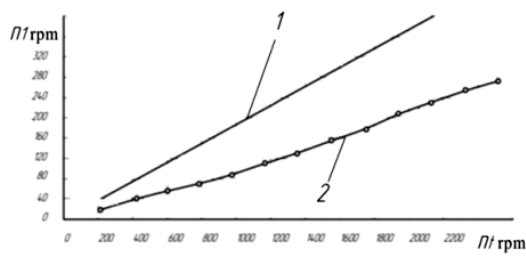

a

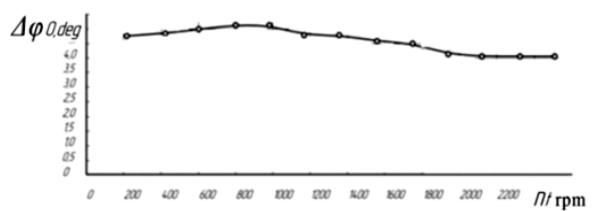

b

Fig. 4. Dependence of the kinematic backlash on the angular velocity of the transmission drive shaft. a) the angular velocity of the output end of the torsion shaft; b) the value of the 'lost' oscillation amplitude $\Delta \varphi$ of the rectifier rocker arms; 1 - theory; 2 - experiment.

The kinematic backlash will be taken into account by introducing the concept of 'kinematic efficiency':

$$
\eta_{k}=\left(\varphi_{0}-\Delta \varphi\right) / \varphi_{0}=1-\Delta \varphi / \varphi_{0}
$$

A similar approach is used in [15]. The value of the 'kinematic efficiency' affects the size of the rectifier free play and the moment of drag of the FWM friction discs [16] (even in the 'stop' mode) and, accordingly, the energy consumption in the transmission, which should be taken into account in the calculations of efficiency.

\section{Calculation of the Blagonravov transmission efficiency}

Here we assume that the efficiency $(\eta)$ is the ratio of the useful work to the supplied work. Then the efficiency can be given as

$$
\eta=\left(A M_{1}^{-}+A M_{2}\right) /\left(A M_{1}^{+}+\left(1-\eta_{0}\right) \Pi+A M_{V}+A M_{K N}\right),
$$

where $A M_{1}^{-}$- the useful work returned by the torsion bar to the transmission drive shaft;

$A M_{2}$ - useful work to cope with the torque resistance $M_{c}$ on the transmission driven shaft (see Fig. 2.);

$A M_{1}^{+}$- work supplied by the ICE when the torsion bar is twisted;

$\left(1-\eta_{0}\right) \Pi$ - work done by the ICE engine to cope with the hysteresis losses in the rectifier's cycle 'loading - unloading';

$\eta_{0}$ - efficiency of the 'rectifier - torsion shaft' complex in the 'stop' mode;

$\Pi$ - maximum potential energy of the twisted torsion shaft; $\Pi=c_{t}\left(\varphi_{t}^{\max }\right)^{2} / 2$, determined in accordance with [3] by the formula

$$
\varphi_{t}^{\max }=\varphi_{0}\left\{2\left(1-i_{t}^{2}\right)^{0,5}-i_{t}\left[\pi-2 \arcsin \left(i_{t}\right)\right]\right\}
$$

$A M_{V}$ - work done by the ICE engine to cope with the moment of drag of the rectifier friction discs while taking up the free play; 
$A M_{K N}$ - work done by the ICE engine to cope with losses in kinematic pairs and oil pump drive [14].

As shown in [14], the transmission efficiency in the 'stop' mode is determined by the empirical formula

$$
\eta_{0}=1-\left[\left(\Delta_{M C X}\right)^{n+1}-\left(\Delta_{M C X}+c\right)^{n+1}\right] /\left[\left(\Delta_{M C X}\right)^{n+1}+0,5(n+1) c_{t} \Delta_{t}^{2} / a\right]
$$

Where $a, n$ and $c$ - coefficients determined for the rectifier and torsion bar by approximating the experimental data [14], $c=-0,141, n=2,580, a=86,661$;

$c_{t}-$ stiffness of the experimental torsion shaft, $c_{t}=66,247 \mathrm{Nm} / \mathrm{deg} ; \Delta_{M C X}-$ angular deformation of the FWM at unspecified load, deg;

$\Delta$ - angular deformation of the torsion bar at unspecified load, deg.

The angular stiffness of the rectifier is a nonlinear function, which is determined by the empirical formula $c_{v}=223,585\left(\Delta_{M C X}\right)^{1,580}$. The angular deformations of the FWM and the torsion bar are determined by simultaneous solution of these equations

$$
\begin{gathered}
\varphi_{t}=\Delta_{M C X}+\Delta_{t} \\
66,247 \Delta_{t}=223,585\left(\Delta_{M C X}\right)^{2,580}
\end{gathered}
$$

In the meantime, the target value is $\varphi_{t}$, determined by reference to (9). Then for the experimental rectifier and the torsion bar the efficiency (17) can be given as

$$
\eta_{0}=1-\left[\left(\Delta_{M C X}\right)^{3,580}-\left(\Delta_{M C X}-0,141\right)^{3,580}\right] /\left[\left(\Delta_{M C X}\right)^{3,580}+1,3683 \Delta_{t}^{2}\right] .
$$

The $A M_{1}^{-}$value is determined by the integration formula $\omega_{1} t_{3} \eta_{k} \varphi_{0} \sin \left(\omega_{1} t\right) M_{2}$ in the limits from $\pi$ to $\omega_{1} t_{3}$ with due account for (8), (9), (10), (11) and (14). The $A M_{2}$ value is determined by integrating the formula $\eta_{k} M_{2}$ in the limits from $\omega_{1} t_{1}$ to $\omega_{1} t_{3}$ with account of (7), (8), (9), (10), (14). The value of $A M_{1}^{+}$is determined by integrating $\eta_{k} \varphi_{0} \sin \left(\omega_{1} t\right) M_{2}$ from $\omega_{1} t_{1}$ to $\pi$ relying on (8), (9), (10), (11), and (14). The work done by the ICE engine to cope with the hysteresis losses in the rectifier is determined taking into account the maximum potential energy of the twisted torsion shaft $\varphi_{t}^{\max }(16)$ and the efficiency in the 'stop' mode (17). The moment of drag $M_{v}[14,16]$ does not depend on the relative angular velocity of the friction discs and is equal to $M_{v}=0,85 \mathrm{Nm}$, as applied to the engineering prototype. Then the work of this moment done per cycle may be calculated as the product of the moment by the sum angle of rotation of the driven friction discs, relative to the driving ones, while taking up the free play. It is determined taking into account the data given in $[9,16](3),(5),(13)$. The $A M_{K N}$ value is calculated by integrating the value $M_{K N}$ $=4 \mathrm{Nm}[14]$ from 0 to $2 \pi$.

As a matter of record, according to (15), the efficiency of the Blagonravov transmission is determined. Figure 5 shows a fragment of the oscillogram of single-load flow [14] in the 'stop' mode as an example. With this oscillogram one can directly determine the experimental value of the efficiency in the 'stop' mode. The ratio of the areas limited by the torque curve on the transmission drive shaft $\left|M_{1}^{-} / M_{1}^{+}\right|$, is proportional to the useful and supplied work and is equal to the transmission efficiency in the 'stop' mode, since the work on the transmission driven shaft at $n_{t}=0$ (see Figure 5) is not performed. The measured value is $\eta^{\ni}=0,76$. Calculations according to the formula (15) have been done with the parameters of the rectifier and the torsion bar used in the experiment: $\varphi_{0}=0.172 \mathrm{rad} ; \varphi_{t}^{\max }$ 
$=0.168 \mathrm{rad}, c_{t}=66.247 \mathrm{Nm} / \mathrm{deg} ; c_{v}=223.585\left(\Delta_{M C X}\right)^{1,580} \mathrm{HMNm} / \mathrm{deg}, \Delta \varphi=0.087 \mathrm{rad}$, $n_{k}=0,494, i_{t}=0, \quad \eta_{0}=0,987, M_{t}=M_{2}=498 \mathrm{Nm}, M_{K N}=4 \mathrm{Nm} \mathrm{[14];} n_{1}=9,64 \mathrm{rpm}$ $\left(\omega_{l}=1,01 \mathrm{rad} / \mathrm{sec}\right) ; M_{v}=0.85 \mathrm{Nm}$. Then according to (15) the calculated transmission efficiency in the 'stop' mode $\eta^{r}=0.853$. The discrepancy between the calculated and measured values is $10.9 \%$.

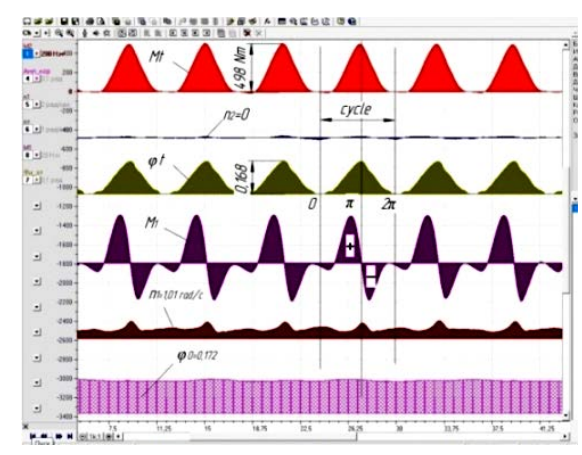

Fig. 5. Fragment of the oscillogram of single-load flow [14] in the 'stop' mode.

\section{Conclusions}

Oscillatory movement of the internal links and the presence of elastic torsion shafts in the continuously variable mechanical transmission make it possible to return part of energy to the drive shaft of the engine, which is considered in the paper as useful. The amount of this recovered energy depends on the transmission operating mode, influenced by the internal gear ratio, and the set oscillation amplitude of the rectifier rocker arm. This leads to the fact that the calculated efficiency of the 'stop mode' is no longer equal to zero. Comparison of measured (efficiency $=0.76$ ) and calculated (efficiency $=0.853$ ) values of transmission efficiency in the 'stop mode' shows that they differ by no more than $11 \%$.

The approach proposed for calculating efficiency provides insight into the operation process of the continuously variable mechanical transmission with oscillatory movements of internal links and force function control.

The research was carried out under the state task No. 0391-2014-007, with partial support from the Institute of Engineering Science of the Russian Academy of Science (Ural Branch). The paper draws upon the materials received within the framework of the grant provided by the Russian Foundation for Basic Research No.16-08-00717 "Developing principles for effective functioning of a new class of continuously variable mechanical transmissions with internal force function", head A. Blagonravov.

\section{References}

1. M. Nagaitsev, S. Kharitonov, E. Yudin, Automatic transmissions in modern passenger cars (M., 2001)

2. I. Kulikov, S. Bakhmutov, L. Lezhnev, J. Mach. Manuf. Reliab., 48, 11 (2019)

3. A. Blagonravov Mechanical continuously variable transmissions (Yekaterinburg, Ural Branch of the Russian Academy of Sciences, 2005)

4. A. Blagonravov, Russ. Eng. Res., 10, 8 (2011) 
5. A. Yurkevich, V. Soldatkin, A. Tereshin, IOP Conf. Ser.: Mater. Sci. Eng, 971, 042018 (2020)

6. A. Blagonravov, A. Yurkevich, V. Soldatkin, A. Tereshin, J. Forestry Russ., 1-2, 9 (2012)

7. A. Leonov, Inertial automatic transformers of a torque (M.: Mashinostroenie Publ., 1978)

8. A. Leonov, A. Dubrovskiy, Mechanical continuously variable transmissions (M.: Mashinostroenie Publ., 1984)

9. A. Yurkevich, J. Adv. Res. Nat. Sci., Issue 8, 38 (2019)

10. A. Blagonravov, E. Revnyakov, J. Automot. Ind., 6, 16 (2008)

11. A. Blagonravov, A.V. Yurkevich, A.A. Yurkevich, J. Automot. Eng., 4, 26 (2015)

12. B. Gasanov, V. Mishustin, Proceedings of Universities, North Caucasian Region, Tech.Sci., 4, 47 (2013)

13. E. Revnyakov, A. Vorontsov, M. Vyaznikov, J. Russ. Eng. Research, 10, 9 (2007)

14. A. Blagonravov, A. Yurkevich, R\&D report, Kurgan, No. PH 01201368139, ICRBS AAAA-B16-21603215007 1-4 (2016)

15. V. Petrov, Automatic control of continuously variable transmissions of self-propelled machines (M: Mechanical Engineering, 1986)

16. V. Soldatkin, A. Tereshin, I. Yurkevich, J. Innov. Sci., 10-2, 116 (2016) 